\title{
Archipel
}

ARCHIPEL Études interdisciplinaires sur le monde insulindien

$101 \mid 2021$

Varia

Monique Zaini-Lajoubert (dir.), L'image de la famille dans les littératures modernes d'Asie du Sud-Est, Paris, Les Indes Savantes, 2019, 326 p. ISBN

978-2-84654-539-6

\section{Elsa Clavé}

\section{OpenEdition}

\section{Journals}

Édition électronique

URL : https://journals.openedition.org/archipel/2509

DOI : 10.4000/archipel.2509

ISSN : 2104-3655

Éditeur

Association Archipel

Édition imprimée

Date de publication : 30 juin 2021

Pagination : 273-275

ISBN : 978-2-910513-85-6

ISSN : 0044-8613

Référence électronique

Elsa Clavé, « Monique Zaini-Lajoubert (dir.), L'image de la famille dans les littératures modernes d'Asie du Sud-Est, Paris, Les Indes Savantes, 2019, 326 p. ISBN 978-2-84654-539-6», Archipel [En ligne], 101 | 2021, mis en ligne le 30 juin 2021, consulté le 02 juillet 2021. URL : http://journals.openedition.org/ archipel/2509; DOI : https://doi.org/10.4000/archipel.2509 
Monique Zaini-Lajoubert (dir.), L'image de la famille dans les littératures modernes d'Asie du Sud-Est, Paris, Les Indes Savantes, 2019, 326 p. ISBN 978-2-84654-539-6

Peu d'ouvrages ont été consacrés, en langue française, aux littératures modernes et contemporaines de l'Asie du Sud-est. Outre le volume pionnier de Pierre-Bernard Lafont et Denys Lombard ${ }^{37}$ (1974), et un premier ouvrage collectif dirigé par Monique Zaini-Lajoubert ${ }^{38}$ (2003) sur le thème de l'émergence des États modernes, nous ne connaissons pas d'ouvrage francophone ayant traité des littératures de la région en profondeur. Le monde anglo-saxon a certes produit un nombre plus important d'études - six au total (p. 12) - mais il convient de noter qu'une partie a pour but premier d'offrir un panorama des littératures de la région, et non une série d'études approfondies des œuvres ${ }^{39}$. À ce titre, l'ouvrage coordonné par Monique ZainiLajoubert forme un apport considérable à notre connaissance de ces littératures.

Dix contributions sur les littératures modernes de sept pays sud-est asiatiques sont regroupées autour du thème de la famille. Ce vaste sujet est abordé dans l'ouvrage sous divers aspects tels le couple traditionnel ou transgressif, la cellule familiale, les rapports aux anciens, ou encore les relations mère-enfants. Les sujets se croisant, les chapitres n'ont pas été organisés thématiquement mais par pays et ordre alphabétique. Les « Relations interpersonnelles en Birmanie » ouvre ainsi l'ouvrage qui se termine par un chapitre sur un récit autobiographique vietnamien. Au total, plus d'une trentaine d'œuvres écrites entre les années 1920 et 2000 - une majorité datant des années 19601990 - sont commentées.

Le couple est naturellement présent dans la quasi-totalité des études mais un chapitre lui est en particulier dédié, celui d'Etienne Naveau, qui analyse la représentation des mariages mixtes dans la littérature de Sumatra ouest entre 1920 et 1950 (p. 83-136). Denise Bernot traite quant à elle des rapports du couple avec le reste de la maisonnée, dont le fonctionnement, et l'éclatement progressif, est au centre d'une nouvelle de l'écrivaine birmane Khin Hnin Yu (p. 25-47). Enfin, dans les deux romans cambodgiens analysés par Suppya Nut, les couples amoureux sont dépeints comme perdants lorsque ceux-ci s'opposent au souhait et à la cohésion de la famille (p. 67-80).

Les rapports entre parents et enfants - en particulier mères et fils - sont également largement représentés. Khamphanh Pravongviengkham aborde, à travers le roman laotien Orage de la vie (1979) de Dao Neua, le thème traditionnel de « la dette du lait maternel » (p. 174) qu'un fils n'a pas eu le temps de rembourser à sa mère en se faisant bonze avant que celle-ci ne décède (p. 157-183). Le roman épistolaire Lettres de Thaïlande (1969), présenté par Jean Baffie, met en lumière les relations d'un immigré chinois avec sa mère, à laquelle il écrit et se confie (p. 209-233). Quant aux

37. Pierre-Bernard Lafont et Denys Lombard (dir.), Littératures contemporaines de l'Asie du Sud-est, Colloque du XXIX ${ }^{e}$ Congrès international des orientalistes, Paris, l'Asiathèque, 1974.

38. Monique Zaini-Lajoubert (dir.), États et littératures en Asie. L'émergence des États modernes, XIX ${ }^{e}-X X^{e}$ siècles, Paris, Les Indes savantes, 2003.

39. Patricia Herbert et Anthony Milner (ed.), Southeast-East Asia : Languages and Literatures : a Select Guide, Honolulu, University of Hawaii Press, 1989; Ulrich E. Kratz (ed.), Southeast Asian Languages and Literatures: A Bibliographic Guide to Burmese, Cambodian, Indonesian, Javanese, Malay, Minangkabau, Thai and Vietnamese, New-York, Tauris Academic Studies, 1996. 
nouvelles thaï contemporaines présentées par Louise Pichard-Bertaux, elles laissent transparaître l'évolution des rapports entre générations en milieu urbain où l'aspect matériel du coût de la vie vient jouer un rôle nouveau au sein de la famille (p. 235256). Plus transgressif, le discours autobiographique de Nguyên Hồng dans " Jours d'enfance », présentée par Doan Cam Thi, évoque le désir sensuel, « dans une intimité des plus évocatrices » (p. 270) d'un fils pour sa mère (p. 259-275).

Mais c'est en fin de compte la place de la femme au sein de la cellule familiale et de la société, que ce soit dans son rôle traditionnel d'épouse, de mère, ou en tant que femme active en dehors du foyer, qui est le plus discuté dans l'ouvrage. Trois chapitres sont en particulier consacrés à ce thème. Sabai Shwe Demaria présente la position de la femme birmane dans la famille à travers le roman Fleur de lotus blanc sur fond d'émeraude (p. 49-63). Monique Zaini-Lajoubert met en lumière la représentation des femmes qui ont, ou pourrait avoir, un rôle en dehors du foyer dans quatre romans malaisiens (p. 187-206), un thème qui est également présent dans le chapitre de Savitri Scherer qui étudie les figures de femmes fortes, au comportement parfois transgressif, dans cinq romans balinais (p. 137-156).

En sus de l'étude des œuvres, plusieurs chapitres présentent le genre littéraire abordé dans son contexte culturel, historique et social, introduisant de ce fait à la littérature du pays. La contribution de Suppya Nut expose ainsi les conditions de l'émergence du roman cambodgien. Jean Baffie traite de la « littérature de langue thaie relative à la vie des Chinois de Thaïlande », donnant la genèse de cette littérature tout en expliquant les raisons de son relatif déclin. Quant à Khamphanh Pravongviengkham, il introduit son étude par des considérations sur la langue et les grandes étapes de la littérature lao. Enfin, on apprend avec Doan Cam Thi comment la conscience de soi, et l'expression des sentiments personnels, a fait son apparition dans le roman vietnamien.

Si l'ouvrage contribue à notre connaissance des littératures modernes et contemporaines de l'Asie du Sud-est, il permet également de prendre conscience des manques de la recherche dans ce domaine. Monique Zaini-Lajoubert précise, en introduction, que la région n'est pas représentée dans sa totalité. Il existe certes une production littéraire inégale d'un pays à l'autre mais c'est surtout le manque d'expertise sur les littératures modernes de cette région qui explique l'absence de certains pays dans cet ouvrage. Bien que Singapour et les Philippines possèdent une importante production littéraire contemporaine multilingue, quasiment aucun travaux n'existe en français sur ces littératures ${ }^{40}$. On regrettera également que la majorité des études adoptent une approche sociologique de ces textes, reléguant les éléments d'analyse littéraire - sur le style et la langue - au second plan. Ils sont pourtant du plus grand intérêt, comme le montre les passages du présent ouvrage (notamment p. 26, $47,70,174,240-241)$, puisqu'ils s'avèrent essentiels pour une approche globale des

40. L'article de Zeus Salazar («Introduction à la littérature moderne tagale », Asian Studies Journal, 11 (1), 1973, p. 129-150) est à notre connaissance la seule étude pour les Philippines. La revue française Jentayu, qui a pour but de faire connaître les littératures d'Asie, a publié les traductions françaises de nouvelles et de poèmes d'auteurs venant de Singapour (numéro spécial Covid-19) et des Philippines (numéros 7 et 10). Deux numéros spéciaux ont par ailleurs été consacrés à la Thaïlande (2017) et l'Indonésie (2018). Louise Pichard-Bertaux et Bernard Sellato, "Autour des littératures contemporaines d'Asie du Sud-Est en France : Thaïlande et Indonésie », Moussons 33, 2019, p. 175-183. 
œuvres, lesquelles gagneraient à être considérées pour leur forme et leur fond. Les évolutions des usages linguistiques font en effet pleinement partie de la "modernité » des littératures que l'on cherche à étudier. On ne peut qu'espérer que l'ouvrage sur l'image de la famille dans les littératures modernes de l'Asie du Sud-est servira d'exemple et inspirera de nombreuses autres initiatives afin de pallier le manque de la recherche actuelle.

Elsa Clavé

Raymond Corbey, Jurookng. Shamanic amulets from Southeast Borneo. Leiden: C. Zwartenkot Art Books, 2018, cartes, photographies, dessins au trait, bibliographie, 243 p. ISBN 978-90-5450-019-3

L'auteur du livre, Raymond Corbey, anthropologue de formation, combine différents talents. Formé à la philosophie et à l'archéologie, il a enseigné à Tilburg et est actuellement rattaché à l'Université de Leyde aux Pays-Bas ${ }^{41}$. Son intérêt pour le statut de l'art tribal en Occident et pour les questions culturelles qui y sont liées a abouti à une approche innovante des collections ethnographiques aux Pays-Bas, associant la tradition historique à une méthodologie comparative et à l'esthétique, qui irrigue ses publications sur les collections muséales et privées.

L'approche choisie par Corbey procède par l'analyse des notions qui relèvent de « l'agentivité » (agency), informant le pouvoir et la matérialité des objets rituels en rapport à la cosmologie. Dans cette perspective, les objets rituels peuvent être considérés comme des «personnes » avec leur arrière-plan d'événements biographiques et de souvenirs qui peuvent agréger matérialité et traces (selon Kopytoff 1986 ; Gell 1998). De sorte que la hiérarchie rituelle est démontrée et légitimée par l'exposition ou l'utilisation des objets, combinée à des effets sociaux et politiques pour s'étendre à un réseau social et de parenté. Le pouvoir est ainsi créé et recréé à mesure qu'il se dégage de ces objets rituels, à des moments cérémoniels-clés. Inversement, il peut refléter une dimension « cachée » contenue dans l'objet du fait qu'il ne représente pas seulement les attributs du chef ou du chamane mais aussi le pouvoir spirituel dans sa capacité à croître. Les objets se trouvant ainsi " animés » par une force dynamique, particulièrement pendant les fêtes ou les rites curatifs, se chargent de sens. Dans un rituel, surtout dans un rituel chamanique, le pouvoir des objets interagit avec les esprits, c'est-à-dire les esprits ancestraux/des morts récents, ceux des esprits bienfaisants et malfaisants que le chamane rencontre durant ses voyages dans les mondes des esprits. Jurookng forme un exemple de cette approche.

Ce livre épais dont le titre, en langue vernaculaire, désigne les amulettes chamaniques des Benua'-Bentian, Tunjung et celles des populations Luangan qui y sont rattachées

\footnotetext{
41. Ses principales publications sont : Tribal Traffic (2000), centré sur les collectionneurs et marchands d'art tribal, Headhunters from the swamps (2010) au sujet du Marind Anim du SudOuest de la Nouvelle Guinée - à savoir la Province de Papua, Indonésie - à partir d'une étude approfondie des archives des Misions de l'Église catholique néerlandaises (Mission du Sacré Cour à Tilburg).
} 